\title{
Editors Introduction to the Special Issue on International Developments in Family Therapy
}

\author{
D. Russell Crane
}

Published online: 28 May 2013

(C) Springer Science+Business Media New York 2013

The purpose of this special issue is to consider the current state of the field in as many areas of the world as possible.

The first goal was to build connections between people. People who share some similar ideas about the importance of family therapy, family involvement in care, or systemic approaches to family support, could look in one location find others of similar interests.

Our second goal was to satisfy a curiosity. We wondered about what is happening in places other than our own. My initial consultations with members of the CoFT Editorial Board confirmed that many of them shared a similar interest and many of them agreed to contribute to the project.

The result is a remarkable collection of ideas, developments, and thinking about how the field "stands" in different places. Forty-seven authors representing four of the six continents (less Africa and Antarctica), who themselves identified nineteen unique (and sometimes overlapping) geographical areas all contributed to this "snapshot" of the field as it appears in the summer of 2013.

The contributors were identified by consulting with members of the CoFT editorial board, gleaning names from the membership lists of different family-based professional organizations, examining the editorial boards of a range of professional journals, and then using the "snowball technique" to identify additional potential authors.

The authors were asked to respond to a framework of topics that included:

"1. History of family therapy in your area including such material as key "founders", or people who began to work in family therapy in your area.

Where and how the early founders received training in family therapy.

Key institutions that began providing services and/or training in family therapy.

A timeline of key developments in your area.

2. How does family therapy fit into the current medical and or social services systems in your area?

D. R. Crane $(\bowtie)$

School of Family Life, Brigham Young University, 257 Taylor Building, Provo, UT 84602, USA

e-mail: russ_crane@byu.edu

URL: http://russcrane.byu.edu 
3. In what contexts (e.g. universities, clinics, colleges, etc.) can one obtain training in systemic therapy? How long is the training? What are the costs of training? What does one receive at the end of training? A university degree, a specialized certificate of completion, or some other formal recognition?

4. What national (or regional) accreditation standards exist for training programs in family therapy?

5. What specialized qualification, licensure, or certification is available for family/ systemic therapy practitioners?

6. In some countries there is a significant overlap between the traditions of family versus couple/marital therapy. In your context how do these fields tend to merge or separate in terms of training and practice?

7. What professional organization(s) are there for family/systemic therapists?

8. Your view of the future directions for family therapy practice, training, and recognition in your area/region.

9. Anything else you would like to add".

Some authors followed the suggested outline closely while others chose a different but equally interesting path.

The order of appearance of the articles does not reflect any ranking by importance or value. Rather, it is the order in which the articles, after review and revision, were accepted "as is" for publication.

Each submission was peer reviewed. However, we did not attempt to compel the authors to use any variant of English at the level of a native speaker. Instead, I wanted the variance in language use to show through, just as variances in culture and regional differences naturally emerge.

We are aware that no single author (or group of authors) can speak definitely for an entire country, region, or area of the world, nor is it possible to provide a single manuscript that covers all aspects of this field. However, we sought a beginning, where interested readers can learn about other contexts that will increase their knowledge about the present, and future of family and systemic therapy.

The project has been successful because of the contributions of many people. First of course, are the authors who were willing to voluntarily share their valuable time and expertise to this unique project. Second are the peer reviewers who also willingly shared their time and talents to make suggestions to improve each submission. Third, my own research team who aided in English language reviews and provided some interesting questions for the authors. Fourth, the support, and encouragement each of us receives from our own families and loved ones that make our work possible. However, the most important contributors are the families we serve. Who through sharing their lives with us, allow us to share our knowledge with others. 